\title{
Will the Mitral Clip become the invasive treatment of choice for hypertrophic obstructive cardiomyopathy?
}

\author{
Mohamad Alkhouli \\ Department of Medicine, Division of Cardiovascular Diseases, Temple University Hospital, Philadelphia, PA, USA
}

Postep Kardiol Inter 2014; 10, 3 (37): 153-154

DOI: 10.5114/pwki.2014.45140

Left ventricular outflow tract obstruction (LVOTO) in classic hypertrophic obstructive cardiomyopathy is caused by a combination of septal hypertrophy and systolic anterior motion of the mitral valve (SAM). Invasive treatment of hypertrophic obstructive cardiomyopathy (HOCM) is reserved for patients with severe drug refractory symptoms and LVOTO, and has historically aimed to reduce the thickness of the basal septum of the left ventricle as a primary cause of LVOTO (septal reduction therapy). Surgical myectomy is highly successful in decreasing LVOTO and considered the invasive therapy of choice for HOCM. Alcohol-induced localised infarction of the basal septum, also known as alcohol septal ablation (ASA), is a percutaneous alternative in poor surgical candidates. When performed by highly experienced operators, surgical myectomy and ASA both have low mortality and high success rates.

Systolic anterior motion of the mitral valve contributes significantly to the development of LVOTO in HOCM patients, and invasive techniques to correct SAM such as mitral valve repair (MVrep) might be as effective as septal reduction therapy in relieving the LVOTO. Until recently, however, MVrep in the treatment of HOCM has been limited to patients undergoing septal myectomy, and have an associated degenerative mitral valve disease. In a large series of HOCM patients undergoing surgical myectomy, only 32 out of 791 patients (4\%) had concomitant MVrep or replacement, and all of those patients had associated degenerative mitral disease [1].

Recent reports of using the Alferi edge-to-edge repair in conjunction with septal myectomy in HOCM patients without degenerative mitral valve disease have shown promising results [2-4]. Since the Alferi technique was only performed concomitantly with septal myectomy, it is difficult to know weather surgical edge-to-edge repair of the mitral valve alone is sufficient to treat HOCM patients with refractory symptoms.
In 2003, a patient with mitral valve prolapse and severe mitral regurgitation was treated with a percutaneous edge-to-edge MVrep using a novel system - the MitraClip. The safety and effectiveness of the MitraClip in treating various causes of mitral regurgitation (MR) is under ongoing investigations. Because SAM of the mitral valve is a major player in the symptomatology of HOCM and patients with this condition usually do not have associated organic mitral valve pathology, it is intuitive to think that the MitraClip can be very effective in treating $M R$ and relieving the LVOTO by reducing or eliminating SAM. However, unfortunately, the published and ongoing trails did not include patients with HOCM. Schafer et al. recently published a very elegant 'proof of concept' series of three patients with LVOTO, who underwent an isolated percutaneous edge-to-edge MVrep using the MitraClip without septal ablation [5]. In this small group of patients the MitraClip showed impressive reduction in LVOT gradient both at rest $(36.6 \pm 34.0 \mathrm{~mm} \mathrm{Hg}$ pre MitraClip vs. 10 $\pm 4.3 \mathrm{~mm} \mathrm{Hg}$ post MitraClip), and after an extra-systolic beat $(123.6 \pm 34.2 \mathrm{~mm} \mathrm{Hg}$ pre MitraClip vs. $21.6 \pm 5.7$ post MitraClip). The procedure resulted in a significant reduction in MR grade from $2.6 \pm 0.5$ to $0.25 \pm 0.35$, and a dramatic improvement of symptoms.

In summary, percutaneous edge-to-edge MVrep is a promising technique in its early stages. The MitraClip and other devices under development might be safe, effective, and attractive 'mini-invasives' treatment modalities in HOCM patients with severe refractory symptoms and persistent LVOT gradient.

\section{References}

1. Wan CK, Dearani JA, Sundt TM 3rd, et al. What is the best surgical treatment for obstructive hypertrophic cardiomyopathy and degenerative mitral regurgitation? Ann Thorac Surg 2009; 88: 727-31.

2. Bhudia SK, McCarthy PM, Smedira NG, et al. Edge-to-edge (Alfieri) mitral repair: results in diverse clinical settings. Ann Thorac Surg 2004; 77: 1598-606.

\section{Corresponding author:}

Mohamad Alkhouli MD, Division of Cardiovascular Diseases, Temple University School of Medicine, 3401 North Broad Street (9 PP), Philadelphia, PA 19140, USA, phone: 215316 0232, fax: 215707 8817, e-mail: adnanalkhouli@gmail.com

Received: 6.07.2014, accepted: 18.07.2014. 
3. Gillinov AM, Smedira NG, Shiota T. Use of the Alfieri edge-to-edge technique to eliminate left ventricular outflow tract obstruction caused by mitral systolic anterior motion. Ann Thorac Surg 2004; 78: e92-3.

4. Tsutsumi Y, Numata S, Seo H, Ohashi H. Transaortic edge-to-edge mitral valve repair and left ventricular myectomy. Gen Thorac Cardiovasc Surg 2013; 61: 223-5.

5. Schäfer U, Frerker C, Thielsen T, et al. MitraClip therapy to reduce left ventricular outflow tract obstruction in hypertrophic obstructed cardiomyopathy. Clin Res Cardiol 2013; 102: 1. 